\title{
Chronic myeloid leukaemia following repeated exposure to chest radiography and computed tomography in a patient with pneumothorax: A case report and literature review
}

\author{
FANG-HE JU ${ }^{1}$, XU-BO GONG ${ }^{2}$, LI-BIN JIANG ${ }^{1}$, HUI-HUA HONG ${ }^{1}$, \\ JUN-CHAO YANG ${ }^{1}$, TING-ZHEN XU ${ }^{1}$, YU CHEN ${ }^{1}$ and ZHEN WANG ${ }^{1}$ \\ ${ }^{1}$ Department of Respiratory Medicine, Zhejiang Provincial Hospital of Traditional Chinese Medicine, \\ Hangzhou, Zhejiang 310006; ${ }^{2}$ Department of Clinical Laboratory, The Second Affiliated Hospital, \\ School of Medicine, Zhejiang University, Hangzhou, Zhejiang 310009, P.R. China
}

Received December 18, 2014; Accepted January 21, 2016

DOI: $10.3892 / \mathrm{ol} .2016 .4236$

\begin{abstract}
It is well known that radioactive rays may cause damage to the human body. Progress in modern medicine has led to an increased risk of therapeutic and diagnostic radiation exposure of patients. Although clear evidence of a radiation dose-dependent risk of chronic myeloid leukaemia, particularly for patients exposed to radiation at a young age, has been established, it is not known whether radiation exposure during diagnostic imaging also increases the risk of cancer. The present study reports the case of a patient who underwent several diagnostic imaging tests (including repeated chest radiography and computed tomography) for recurrent pneumothorax. At around one year subsequent to these tests, the patient was diagnosed with chronic myeloid leukaemia. The patient exhibited an increase in white blood cell count over time, and a bone marrow smear test showed a myeloid/erythroid ratio of 13.9:1. In addition, the qualitative breakpoint cluster region (BCR)/Abelson (ABL) gene test revealed positive results for BCR/ABL fusion (p210). Based on the data reported in the current case, research aimed at elucidating the potential risks associated with diagnostic radiation is urgently required. It is crucial that medical professionals consider the potential harmful side effects of diagnostic radiation when ordering radiation-based diagnostic imaging examinations.
\end{abstract}

Correspondence to: Dr Fang-He Ju or Dr Zhen Wang, Department of Respiratory Medicine, Zhejiang Provincial Hospital of Traditional Chinese Medicine, 54 Youdian Road, Hangzhou, Zhejiang 310006, P.R. China

E-mail: jufanghe2001@126.com

E-mail: wangzhen610@sina.cn

Key words: leukaemia, pneumothorax, tomography, radiography, case report, review

\section{Introduction}

Radioactive rays are known to damage the human body, causing conditions such as malignant tumours. Studies have demonstrated that the exposure of healthy tissue to harmful radiation during radiation therapy may lead to an acute radiation reaction or cancer, particularly leukaemia (1). Clear evidence of a dose-dependent risk of chronic myeloid leukaemia (CML), especially in people exposed to radiation at a young age, has been reported (2), and numerous studies on the association between radiotherapy and CML have been published (3-5).

CML is characterized by the nonrandom, recurrent Philadelphia chromosome (6), which is present in $~ 90 \%$ of patients with CML (7). Philadelphia chromosome carries the breakpoint cluster/Abelson murine leukemia viral oncogene homolog 1 fusion gene, which has been demonstrated to play a major role in CML pathogenesis $(8,9)$. The incidence of CML is $\sim 0.36$ out of every 100,000 individuals in China (10). In recent years, more effective techniques have been introduced as therapy for patients with CML, including the use of interferon- $\alpha$, allogeneic stem cell transplantation and tyrosine kinase inhibitors (11). Currently, the prognosis of patients CML is markedly improved compared to previous years. A previous study, which used imatinib as a first-line treatment for newly diagnosed patients with chronic phase CML, demonstrated that the likelihood of succumbing to CML was almost equivalent to the probability of succumbing to other diseases (12).

It is unclear, however, whether diagnostic radiation increases the risk of CML, the incidence of which is $\sim 0.36$ out of every 100,00 individuals in China. Case-control and retrospective studies that have investigated the risk of developing CML in patients exposed to diagnostic radiation have resulted in contradictory data (13-15). The reported odds ratios for diagnostic radiation-induced CML vary greatly, possibly due to the different dosages of radiation exposure used in the different studies. To the best of our knowledge, only one case of possible diagnostic radiation-induced CML exists in the literature (13). Thus, robust evidence to support the association between diagnostic radiation and CML is still lacking. The present study reports the case of a patient who developed CML 


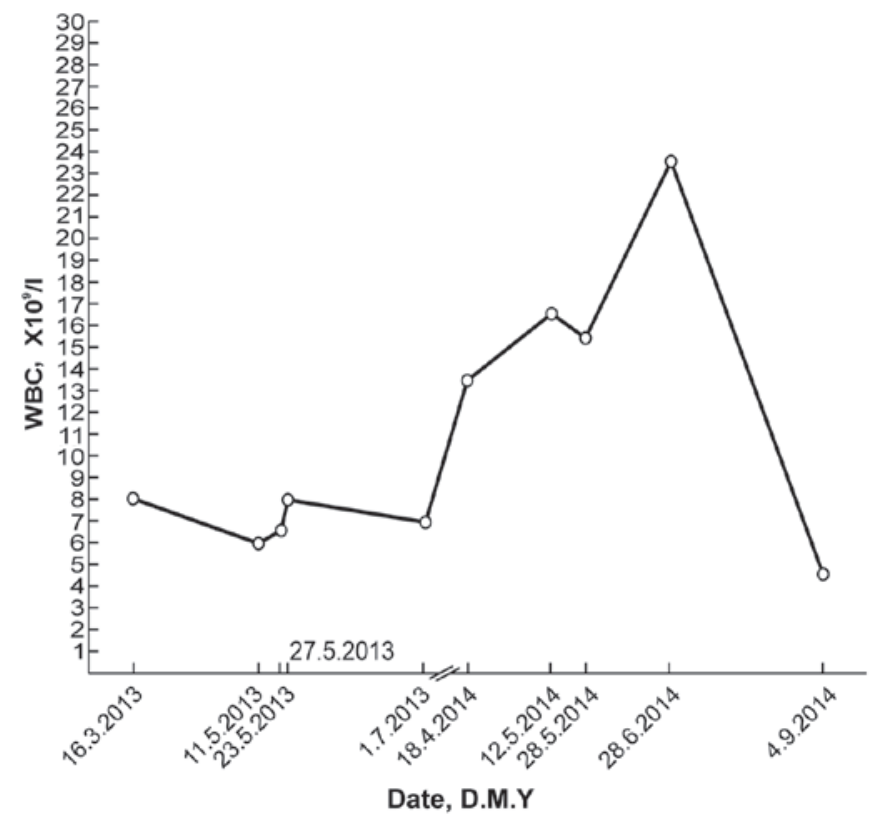

Figure 1. Changes in the patient's WBC count over time. WBC, white blood cell; D.M.Y., day/month/year.

subsequent to undergoing several diagnostic imaging tests for recurrent pneumothorax.

\section{Case report}

On 6 May 2013, a 26-year-old man presented to the Zhejiang Provincial Hospital of Traditional Chinese Medicine (Hangzhou, China) with pneumothorax. The patient eventually recovered from the condition on 3 June 2013; however, between 6 May 2013 and 3 June 2013, the patient was admitted as an in-patient to the Zhejiang Provincial Hospital of Traditional Chinese Medicine (Hangzhou, China) and the Linyi People's Hospital (Linyi, China) on three occasions due to recurrent pneumothorax. He was treated with closed thoracic drainage and underwent thoracoscopic surgery and Bullae resection due to a relapse. Routine blood tests, bowel and urine output, liver and kidney function, and tumour markers were all normal. Viral hepatitis and human immunodeficiency virus infection were also ruled out during the hospital visit.

During the first month of treatment (from the day of the first occurrence of pneumothorax to the date of discharge subsequent to surgery), the patient was exposed to eight chest radiography examinations, comprising eight exposures in the anteroposterior and two in the posteroanterior position. Additionally, three chest computed tomography (CT) scans of the entire body were performed at various hospitals. The effective radiation dose of these imaging tests reached $9.6 \mathrm{mSv}$ (16). Within the next 8 months, the patient underwent two more chest radiography procedures (two exposures in the anteroposterior and one in the posteroanterior position) and one CT scan due to a cough and other discomfort. These added an additional radiation dose of $2.1 \mathrm{mSv}$, yielding a total effective dose of $11.7 \mathrm{mSv}$.

The patient was an employee of a large-scale bookstore and lived in a normal environment, which had no serious pollution, abnormal background radiation or other anomalies. He denied

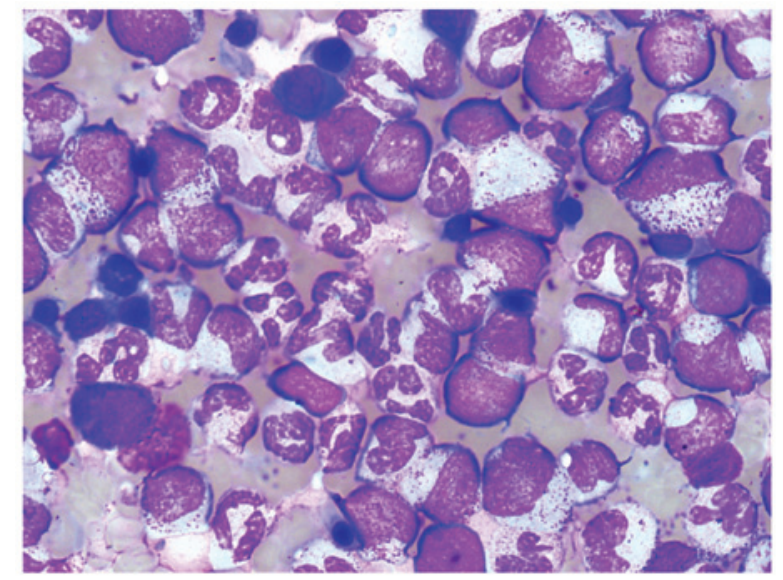

Figure 2. Bone marrow smear with Wright-Giemsa staining viewed under oil immersion lens, revealing bone marrow hematopoietic hyperactivity, primarily with neutrophils. Magnification, x1,000.

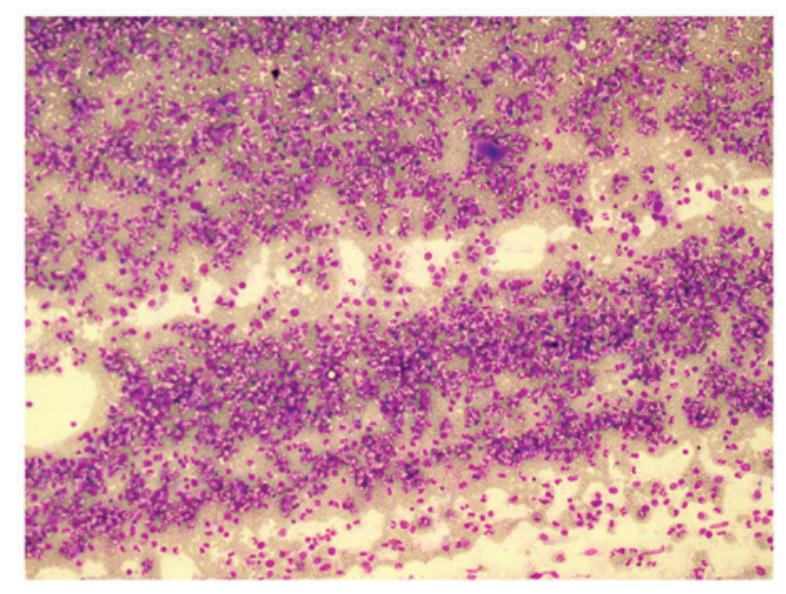

Figure 3. Bone marrow smear. Wright-Giemsa staining was used to visualize bone marrow cells, revealing an abnormal increase in bone marrow nucleated cells. Magnification, x100.

exposure to toxic substances or contaminated water, alcohol abuse or exposure to venereal disease. The patient's family histories were normal, and none of his immediate family members had cancer. The patient was slim and had a body mass index of $20.2 \mathrm{~kg} / \mathrm{m}^{2}$. Physical examination revealed no remarkable findings.

A routine blood test on 18 April 2014, during a general health examination, revealed a white blood cell (WBC) count of $13.9 \times 10^{9} / 1$ (normal range, $4-10 \times 10^{9} / 1$ ) with $66.9 \%$ neutrophils (normal range, 50-70\%), a red blood cell (RBC) count of $5.25 \times 10^{12} / 1$ (normal range, $4.5-5.5 \times 10^{12} / 1$ ) and a platelet (PLT) count of $335 \times 10^{9} / 1$ (normal range, $100-300 \times 10^{9} / 1$ ). The patient's WBC count subsequently increased significantly (Fig. 1). A bone marrow smear test, stained with Wright-Giemsa (Zhuhai Baso Biotechnology Co., Ltd., Zhuhai, China), was performed on 13 June 2014 and revealed bone marrow hematopoietic hyperactivity primarily with neutrophils (Fig. 2) and an abnormal increase in bone marrow nucleated cells (Fig. 3). Bone marrow biopsy core from the patient was fixed using Bouins fixative solution (Sigma-Aldrich, St. Louis, MO, USA), dehydrated using ethanol, plastic embedded (Hemapun 959; 


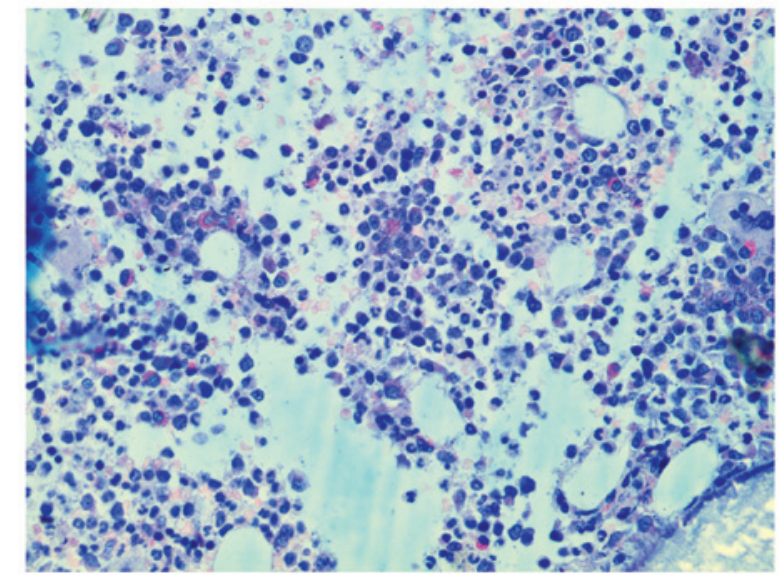

Figure 4. Histological examination of the bone marrow. Improved toluidine blue staining was used to visualize bone marrow cells, revealing an increased number of positive basophils (dyed red). Magnification, x400.

Beijing Xinxing Braim Technology Co., Ltd., Beijing, China), sectioned $(3-\mu \mathrm{m})$ using a microtome (Microm HM340E; Microm International GmbH, Walldorf, Germany) and stained with hematoxylin and eosin, hematoxylin-Giemsa-fuchsin, Giemsa, improved toluidine blue, periodic acid Schiff reagent and Gomori silver impregnation to identify reticulin and collagen fibers (Zhuhai Baso Biotechnology Co., Ltd.). Histological examination of the bone marrow using toluidine blue staining demonstrated an increased number of positively-stained basophils (Fig. 4), and a blood smear test revealed multiple circulating immature granulocytes. A chromosome karyotype analysis (19 June 2014) revealed the following results: 46,XY,t(9;22)(q34;q11.2). The qualitative breakpoint cluster region (BCR)/Abelson (ABL) gene test revealed positive results for $B C R / A B L$ p210 and negative results for BCR/ABL p230. The negative control group yielded negative results and the positive and internal control groups showed positive results. All these results suggested CML. Hence, a diagnosis of CML was made.

A repeat routine blood test (28 June 2014) revealed a WBC count of $23.8 \times 10^{9} / 1$ with $73.8 \%$ neutrophils, an RBC count of $4.89 \times 10^{12} / 1$ and a PLT count of $507 \times 10^{9} / 1$. Following the administration of oral imatinib mesylate ( $400 \mathrm{mg} / \mathrm{d}$ for 68 days), the patient's leukocyte percentage decreased to normal levels. Another repeat routine blood test (4 September 2014) revealed a WBC count of $4.6 \times 10^{9} / 1$ with $45.5 \%$ neutrophils, an RBC count of $3.72 \times 10^{12} / 1$ and a PLT count of $212 \times 10^{9} / 1$. Changes in the patient's WBC values over time are depicted in Fig. 1. The patient continued to take imatinib mesylate until January 2015 , and experienced no obvious discomfort. The patient remains in follow-up, and will undergo bone marrow cytology and chromosome examination.

The institutional ethics committee of the Zhejiang Provincial Hospital of Traditional Chinese Medicine approved the current study.

\section{Discussion}

The current study reports a case of CML occurring in a patient who had undergone repeated exposure to diagnostic chest radiography and $\mathrm{CT}$ for pneumothorax. The patient appeared irritable and slightly anxious during hospital visits. He had been a patient of the hospital three times within one month due to his pneumothorax. Irrespective of how he felt, he requested a radiological examination (chest radiography or CT). Furthermore, the patient always appeared to be in a hurry and was anxious to achieve recovery in order to be discharged from the hospital. These factors contributed to the decision of the doctors to perform multiple chest radiography inspections in a fairly short space of time.

The International Commission on Radiological Protection (ICRP) recommends an effective maximum dose of occupational X-ray exposure (in planned exposure situations) of $20 \mathrm{mSv} /$ year when averaged over defined 5-year periods (100 mSv in 5 years) (17). Additionally, the effective dose should not exceed $50 \mathrm{mSv}$ in any single year. However, the ICRP guidelines should not be applied to individual patients, as they may reduce the effectiveness of the patient's diagnosis or treatment, and should only be applied to personnel engaged in radiation-associated industries (16). In consideration of a patient's anxiety levels and to determine the causes and courses of their illnesses, physicians often order numerous chest radiography and CT procedures, as per the ICRP recommendation cited above. As a result, patients frequently have repeated exposure to diagnostic chest X-rays and CT radiation. The dose the present patient received was $<20 \mathrm{mSv}$; however, the patient was diagnosed with CML. Therefore, even though the guidelines of the ICRP recommends not to exceed $50 \mathrm{mSv}$, occasionally a lower dose may cause harm, which radiologists must be made aware.

Although a number of case-control and retrospective studies have investigated the risk of CML in patients exposed to diagnostic radiation, their results have been contradictory (13-15). One limitation of previous case-control studies of leukaemia and its association with diagnostic radiography was the lack of dosimetry. Evans et al (18) applied dose-response models to new data on population exposure to radiographic procedures during a 1-year period, and concluded that $1 \%$ of all leukaemia cases were caused by diagnostic radiography. However, this study had limited information on repeat examinations. To the best of our knowledge, only a single case report presenting a possible cases of diagnostic radiation-induced CML exists (13). One report stated that a 19-year-old man with Ollier's disease who underwent multiple orthopaedic procedures for leg length discrepancy developed CML presenting with intramuscular haematoma. In this case, the cumulative X-ray exposure of repeated surgeries between the ages of 7 and 12 years was estimated to be around $16 \mathrm{mSv}$, which approximately equals the radiation dose of 720 chest radiography procedures. The authors speculated that the repeated radiation exposure, particularly due to the patient's young age and the exposure of the marrow tissue of the long bones to radiation, may have been an important pathogenetic factor for CML (13). The current case is different from this case in numerous aspects. Firstly, although the patients were both male, the patient in the present report was exposed to radiation as an adult and within a shorter time frame. Additionally, the radiation dosage in the current patient was lower, and the chest was exposed to radiation, rather than the long bones as in the previous case. The patient in the case report by $\mathrm{Au}$ et al (13) possessed a Philadelphia translocation, $\mathrm{t}(9 ; 22 ; 13)(\mathrm{q} 34 ; \mathrm{q} 11.2 ; \mathrm{q} 12)$, with a predominance of neutrophil 
elastase (ela2) BCR/ABL splicing and deletion of the reciprocal der(9) ABL/BCR locus. In the present case, the qualitative $\mathrm{BCR} / \mathrm{ABL}$ fusion gene (major breakpoint) test performed on 19 June 2014 revealed positive results for BCR/ABL p210 and negative results for BCR/ABL p230. Collectively, these findings make the current case unique.

Cases of leukaemia occurring following the receipt of radiological examinations have rarely been reported. A survey by Sodickson et al (19) conducted on 31,462 patients receiving CT examinations during 2007 assessed the lifetime attributable risks of radiation-induced cancer from the cumulative radiation dosage using the reported biological effect of ionizing radiation, based on the gender and age of the study participants. The results revealed that $\mathrm{CT}$ radiation dosage that accumulated over time could raise the baseline for cancer risk in this patient population. The majority of patients, however, exhibited a low risk of being diagnosed with a radiation-induced tumour, and only a small number of patients were at a potentially higher risk for cancer due to frequent CT scans (19). It has been suggested that this discrepancy could be associated with the patients' individual differences in susceptibility to radiation. Indeed, the ICRP has suggested that patients who are carriers of cancer genes may be at risk of developing tumours as a result of radiation therapy. In particular, in those who are already suffering from cancer, radiation therapy may risk triggering a secondary tumour (20). As stated on page 143 of the ICRP Publication 103 (17), 'including genetic susceptibility of strongly expressed genes to radiation-induced cancer that are considered to be rare, which is unlikely to cause any significant distortion to risk assessment of the population; and the potential impact of the often seen weakly expressed genes is still unknown'. Furthermore, the authors state that 'strongly expressed, highly susceptible cancer genes are rarely seen. On this part, the report made the low dose radiation cancer risk assessment based on population as a whole, which should not be enough to cause significant distortions' (16).

Other studies have demonstrated that the repair capacity or fidelity of DNA and radiation sensitivity are associated with racial diversity in humans (21). Studies have also reported that radiation susceptibility causing increased cancer risk is polygenic in nature. Radiation susceptibility- and normal tissue toxicity-related genes and pathways include those for sensing DNA damage, cell cycle checkpoints, intermediate protein recruitment, repair pathways (base excision, homologous recombination and non-homologous end joining), apoptosis, inflammatory cytokines, fibrosis proteins, extracellular matrix, antioxidant enzymes, cytokines, and growth factors (22). However, it is still unknown whether individual sensitivity to cancer caused by radiation actually exists, and if there is a causal relationship between diagnostic radiation and cancer (21). The issue of individual susceptibility to genetic damage caused by diagnostic radiation is a cause for concern and worthy of further discussion.

In clinical practice, making a diagnosis commonly requires multiple radiological examinations or treatments. Although it is not possible to be sure what role the current patient's radiological examination played in his CML diagnosis, the case data presented in this report are intended to increase awareness of the potential harm of diagnostic radiation. Future in-depth studies aimed at understanding individual susceptibility to the effects of diagnostic radiation damage are warranted.

\section{Acknowledgements}

The authors wish to thank Editage (www.editage.cn/) for English language editing, and the National Natural Science Foundation of China (Beijing, China; grant no., 81400107) for supporting the present study.

\section{References}

1. Hall EJ and Giaccia AJ: Radiobiology for the Radiologist. 7th edition. Philadelphia: Wolters Kluwer Health/Lippincott Williams \& Wilkins: 273-302, 2012.

2. Tsushima H, Iwanaga M and Miyazaki Y: Late effect of atomic bomb radiation on myeloid disorders: Leukemia and myelodysplastic syndromes. Int J Hematol 95: 232-238, 2012.

3. Ural AU, Beyzadeoglu M, Avcu F and Nevruz O: Chronic myeloid leukemia following radiotherapy for carcinoma of the cervix: Report of a case and brief review of the literature. Am J Hematol 82: 415-416, 2007.

4. Shimon I, Kneller A and Olchovsky D: Chronic myeloid leukaemia following 131I treatment for thyroid carcinoma: A report of two cases and review of the literature. Clin Endocrinol (Oxf) 43: 651-654, 1995.

5. Aguiar RC: Therapy-related chronic myeloid leukemia: An epidemiological, clinical and pathogenetic appraisal. Leuk Lymphoma 29: 17-26, 1998.

6. Nowell PC and Hungerford DA:s A minute chromosome in human chronic granulocytic leukemia. Science 132: 1497-1501, 1960.

7. Hochhaus A: Detection and quantification of leukemia-specific rearrangements. In: Methods in Molecular Medicine $\mathrm{T}^{\mathrm{TM}}$. Molecular Analysis of Cancer. Boultwood J and Fidler C (eds). Humana Press, Totowa, NJ, pp67-96, 2001.

8. Konopka JB, Watanabe SM, Singer JW, Collins SJ and Witte ON: Cell lines and clinical isolates derived from $\mathrm{Ph} 1-$ positive chronic myelogenous leukemia patients express c-abl proteins with a common structural alteration. Proc Natl Acad Sci USA 82: 1810-1814, 1985.

9. Davis RL, Konopka JB and Witte ON: Activation of the c-abl oncogene by viral transduction or chromosomal translocation generates altered c-abl proteins with similar in vitro kinase properties. Mol Cell Biol 5: 204-213, 1985.

10. Wang JY, Liao EY, Hu PJ: Leukemia. In: Internal Medicine. People's Medical Publishing House, Bejing, pp756-757, 2005 (In Chinese).

11. Hughes T, Deininger M, Hochhaus A, Branford S, Radich J, Kaeda J, Baccarani M, Cortes J, Cross NC, Druker BJ, et al: Monitoring CML patients respongding to treatment with tyrosine kinase inhibitors:review and recommendations for harmonizing current methodology for detecting BCR-ABL transcripts and kinase domain mutations and for expressing results.Blood 108: 28-37, 2006.

12. Shah NP: Front-line TKI therapy for chronic-phase CML: The luxury of choice. Oncology (Williston Park) 26: 908, 910, 912, 2012.

13. Au WY, Ooi GC, Ma SK, Wan TS and Kwong YL: Chronic myeloid leukemia in an adolescent with Ollier's disease after intensive X-ray exposure. Leuk Lymphoma 45: 613-616, 2004.

14. Yuasa H, Hamajima N, Ueda R, Ohno R, Asou N, Utsunomiya A, Ogura M, Takigawa N, Ueda T, Hiraoka A, et al: Case-control study of leukemia and diagnostic radiation exposure. Int J Hematol 65: 251-261, 1997.

15. Preston-Martin S, Thomas DC, Yu MC and Henderson BE: Diagnostic radiography as a risk factor for chronic myeloid and monocytic leukaemia (CML). Br J Cancer 59: 639-644, 1989.

16. United Nations Scientific Committee on the Effects of Atomic Radiation: Effects of ionizing radiation. In: UNSCEAR 2006 report. Report to the general assembly, with scientific annexes. Annex A: Epidemiological studies of radiation and cancer. United Nations, New York, pp13-322, 2006.

17. ICRP: The 2007 Recommendations of the International Commission on Radiological Protection. ICRP Publication 103. Ann ICRP 37 (2-4), 2007.

18. Evans JS, Wennberg JE and McNeil BJ: The influence of diagnostic radiography on the incidence of breast cancer and leukemia. N Eng J Med 315: 810-815, 1986. 
19. Sodickson A, Baeyens PF, Andriole KP, Prevedello LM, Nawfel RD, Hanson R and Khorasani R: Recurrent CT, cumulative radiation exposure, and associated radiation-induced cancer risks from CT of adults. Radiology 251: 175-184, 2009.

20. United Nations Scientific Committee on the Effects of Atomic Radiation. Sources and effects of ionizing radiation. In: UNSCEAR 1993 report. Report to the general assembly, with scientific annexes. Annex F: Influence of dose and dose rate on stochastic effects of radiation. New York: United Nations 619-728, 1993.
21. Sigurdson AJ and Stram DO: Genetic predisposition to radiation-related cancer and potential implications for risk assessment. Ann ICRP 41: 108-116, 2012.

22. Barnett GC, West CM, Dunning AM, Elliott RM, Coles CE, Pharoah PD and Burnet NG: Normal tissue reactions to radiotherapy: Towards tailoring treatment dose by genotype. Nat Rev Cancer 9: 134-142, 2009. 